\title{
Quantitative control of protein and cell interaction with nanostructured surfaces
}

\section{by cluster assembling}

Carsten Schulte, Alessandro Podestà, Cristina Lenardi, Gabriella Tedeschi and Paolo Milani*

${ }^{1}$ CIMAINA e Dipartimento di Fisica, Università degli Studi di Milano, via Celoria 16, 20133 Milano, Italy. ${ }^{2}$ Dipartimento di Medicina Veterinaria, Università degli Studi di Milano, via Celoria 10, 20133 Milano, Italy.

\section{CONS P E C T US}

The development of smart prosthetics, scaffolds and biomaterials for tissue engineering and organ-on-a-chip devices heavily depends on the understanding and control of biotic/abiotic interfaces. In recent years the nanometer scale emerged as the predominant dimension for processes impacting on protein adsorption and cellular responses on surfaces. In this context the extracellular matrix (ECM) can be seen as the prototype for an intricate natural structure assembled by nanoscale building blocks forming highly variable nanoscale configurations, dictating cellular behavior and fate. How exactly the ECM nanotopography influences mechanotransduction, i.e. the cellular capacity to convert information received from the ECM into appropriate responses, remains partially understood, due to the complexity of the involved biological structures, limiting also the attempts to artificially reproduce the nanoscale complexity of the ECM. In this Account we describe and discuss our strategies for the development of an efficient and large-scale bottom-up approach to fabricate surfaces with multiscale controlled disorder as substrates to study quantitatively the effect of nanoscale topography on biological entities. Our method is based on the use of supersonic cluster beam deposition (SCBD) to assemble, on a substrate, neutral clusters produced in the gas phase and accelerated by a supersonic expansion. The assembling of clusters in the ballistic deposition regime follows simple scaling laws, allowing the quantitative control of surface roughness and asperity layout over large areas. Due to their biocompatibility, we focused on transition metal oxides nanostructured surfaces assembled by titania and zirconia clusters. We demonstrated the engineering of structural and functional properties of the cluster-assembled surfaces with high relevance for the interactions at the biotic/abiotic interface. We observed that isoelectric point and wettability, crucial parameters for the adhesion of biological entities on surfaces, are strongly influenced and controlled by the nanoscale roughness. By developing a high-throughput method (Protein Surface Interaction Microarray- PSIM) we characterized quantitatively the capacity of the nanostructured surfaces to adsorb proteins, showing that with increasing roughness the adsorption rises beyond what could be expected by the increase in specific area, paralleled by an almost linear decrease in protein binding affinity. We also determined that the spatial layout of the surface asperities effectively perceived by the cells mimics at the nanoscale the topographical ECM characteristics. The interaction with these features consequentially regulates parameters significant for cell adhesion and mechanotransductive signaling, such as integrin clustering, focal adhesion maturation and the correlated cellular mechanobiology, eventually impacting on the cellular program and differentiation, as we specifically showed for neuronal cells. 


\section{INTRODUCTION}

The understanding of the mechanisms by which biological entities such as proteins and cells interact individually and collectively with artificial surfaces, requires the capability of dissecting complex environments organized over different structural and temporal length scales. ${ }^{1-3}$ This is of fundamental importance for the rational design of biotic/abiotic interfaces enabling novel drug delivery strategies, ${ }^{4}$ fabrication of artificial organs and smart prosthetics. ${ }^{1-5}$

In the last two decades increasing attention has been focused on the nanometer scale as a length scale with fundamental influence on the cellular response and organization. ${ }^{1-3}$ This has stimulated the emergence of a paradigm that recognizes the natural extracellular matrix (ECM) as the prototype of a structure made by nanoscale building blocks whose hierarchical organization influences and determines the cell fate. ${ }^{2,5}$ The reproduction of this multiscale structure on artificial surfaces is thus considered as necessary for the fabrication of realistic biotic/abiotic interfaces. ${ }^{5}$

A realistic model recapitulating the ECM complexity should be based on the 3D organization of biochemical and topographical cues as an essential aspect regulating cellular functions. Cell morphology, motility, and differentiation potential are expected to be profoundly different depending on the dimensionality of their environment. The full control over the properties of artificial 3D structures mimicking ECMs is very hard to achieve, and the fabrication of 3D microenvironments for cell culture is still in its infancy. ${ }^{2,3,5}$

In order to identify the fundamental elements concurring to the ECM topographic complexity and to recognize the structural ingredients for the mimicking of the natural ECM characteristics, a huge effort has been concentrated on a reductionist approach. This consists in the micro and nanofabrication of simple basic 
motifs such as grooves, pillars, dots with different dimensions and pitches, in order to dissect and to recapitulate the elemental topographical cues that may influence the cell behavior. ${ }^{5,6}$ This approach relies on very expensive and difficult to scale-up micro- and nanofabrication techniques (top-down lithographic approaches, micromolding, nanoimprint, etc.). ${ }^{6}$ Beside these limitations, it is yet to be demonstrated that starting from simple topographical motifs one can reconstruct realistically the ECM complexity., ${ }^{3,5}$

On the other hand, methods exploiting chemical or physical etching have been extensively used in the production of implants, since they provide the possibility of obtaining disordered surfaces at the nano- and microscale over large surfaces of different metallic materials at low cost. However, the tuning to the best performing surface topography in terms of cell adhesion and differentiation is based essentially on a trial-and-error approach, with no predictive quantitative evaluation of the effect of the topographical features. ${ }^{7}$

During the last ten years, we concentrated on the development of an alternative additive method to fabricate surfaces with multiscale controlled disorder as substrates to study quantitatively the effect of nanoscale topography on biological entities. ${ }^{8}$ We used transition metal oxides nanostructured surfaces and, in particular, titania and zirconia surfaces. This choice has been dictated by the biocompatibility of these two materials, and by their widespread use as implant and prosthetic materials. ${ }^{9,10}$ A huge literature exists on the production and characterization of biocompatible titania and zirconia surfaces,${ }^{11}$ however no systematic and quantitative characterization of the role of the nanoscale in determining the interaction of elementary biological entities up to the cell is available. 
Our aim has been the development and assessment of a method able to sufficiently explore the parameter space of topographical cues by the rapid parallel fabrication of surfaces with different nanoscale topographies and subsequent highthroughput assaying of these surfaces exposed to the different conditions that can affect their biological activity. ${ }^{12,13}$

We developed a bottom-up fabrication method based on the use of supersonic cluster beam deposition (SCBD) ${ }^{14,15}$ that consists in the assembling on a substrate of neutral clusters produced by condensation in the gas phase and accelerated by a supersonic expansion. ${ }^{16}$ This approach produces nanostructured films with a nanoscale topography whose roughness can be accurately controlled and varied in a reproducible manner. ${ }^{8,17}$ The extremely precise control over nanoscale topography can be easily obtained over macroscopic areas as is required for the large number of experiments typical of in vitro biological assays. ${ }^{18,19}$

\section{RAPID FABRICATION OF NANOSTRUCTURED BIOCOMPATIBLE} SUBSTRATES WITH CONTROLLED TOPOGRAPHICAL FEATURES

The roughening of surfaces and interfaces resulting from the bottom-up assembling of nanoscale objects (atoms, clusters) has a fundamental relevance for a very broad range of phenomena and technological applications. ${ }^{20}$ Many measurable quantities characterizing the roughening obey simple scaling laws and they can be related to macroscopic parameters of the deposition such as time and system dimensions. $^{20,21}$ This is true for cluster-assembled surfaces having a nanoscale morphology, so that their roughness, porosity and specific surface area can be precisely related to scaling exponents that allow prediction, and control of the evolution of their surface morphology. ${ }^{8,22}$ 
The tool that we have developed for the large-scale production of nanostructured surfaces with controlled morphology is a SCBD apparatus equipped with a Pulsed Micro-plasma Cluster Source (PMCS) ${ }^{14}$ (Figure 1). The fundamental and technical principles of SCBD are described and discussed in details in Refs [ $\left.{ }^{14,23}\right]$.

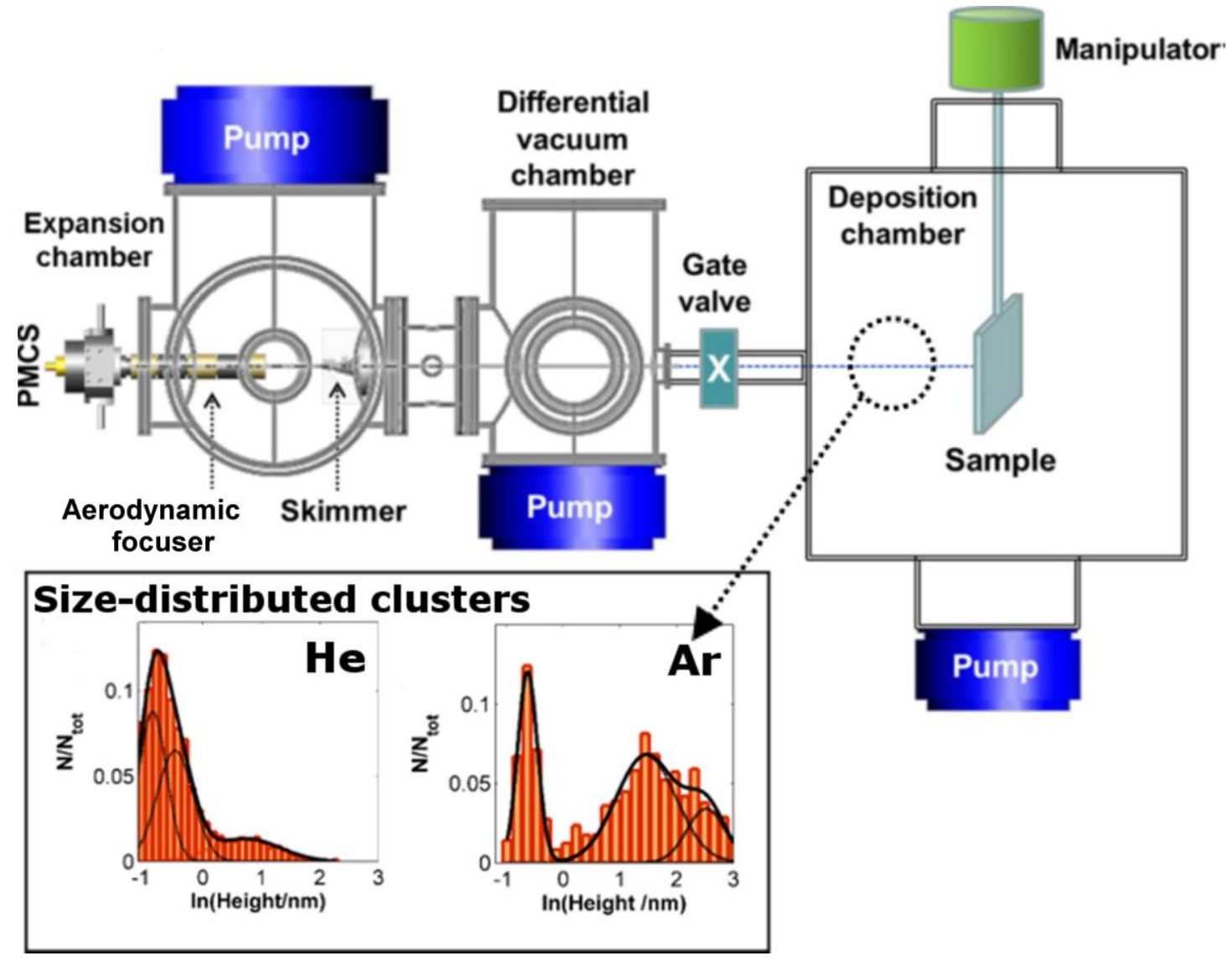

Figure 1. Supersonic Cluster Beam Deposition apparatus. Clusters are formed in the stagnation cavity of the PMCS, where an ionized supersonic beam of Ar or He sputters the target material; the ablated species thermalize with the gas and condense forming clusters. The carrier gas/clusters mixture is accelerated from the PMCS cavity (higher pressure) to the expansion chamber (lower pressure, high vacuum), and collimated through the aerodynamic focuser. Upon selection of the central portion of the beam by a skimmer, nanoparticles enter the deposition chamber and are deposited on a substrate, forming a thin film with thickness in the range 1-1000 $\mathrm{nm}$. In the inset two typical size distributions of titania clusters produced 
using $\mathrm{He}$ and $\mathrm{Ar}$ as carrier gases are shown. Adapted with permission from Ref. [8]. Copyright 2015 AIP Publishing.

The neutral clusters in the supersonic beam possess a rather broad size distribution, which depends on the carrier gas and on the operational parameters of the PMCS. By controlling the working parameters of the cluster source, the aerodynamic filters, and the portion of the beam intercepting the substrate, the size distribution of nanoparticles can be precisely tuned and reproduced (see the inset of Figure 1). Consequently, the deposition time controls the surface morphology of the films, which evolves regularly according to simple and reproducible scaling laws.

We characterized a very large number of samples produced in a time window of approximately 10 years with different SCBD apparatus in academic and industrial facilities. ${ }^{8,17}$ The analysis of surface morphology of cluster-assembled films confirms that the evolution of morphological parameters can be described by the FamilyVicsek scaling relations typical of a variety of growing interfaces, ${ }^{20,21,24-26}$ resulting in a scale invariant nanotopography. The scaling of the RMS roughness w with the thickness $\mathrm{h}$ of nanostructured $\mathrm{TiO}_{2}$ and $\mathrm{ZrO}_{2}$ films obeys the power law $\mathrm{w} \sim \mathrm{h}^{\beta}$, with $\beta=0.37-0.39$ (see Figure 2). Here $w$ is calculated as standard deviation of surface heights on a $2 \mu \mathrm{m}$ scale; $\mathrm{h}$ is measured by scanning the AFM tip across a sharp step on the film produced by masking the substrate during cluster deposition. The values of the growth exponent $\beta$, irrespective to the material deposited, are close to those of the ballistic deposition growth model (for which $\beta=0.33,0.25$ in $1+1$ and $2+1$ dimensions, respectively), where incoming particles land on the growing interface, stick, and do not diffuse significantly. ${ }^{20,21,25,26}$ 


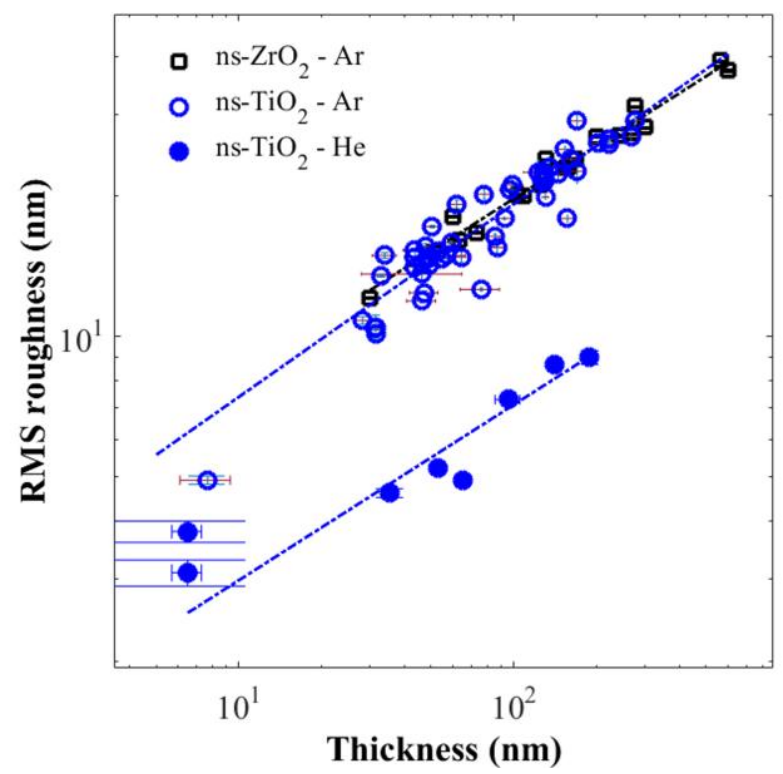

Figure 2. Scaling of surface RMS roughness of ns- $\mathrm{TiO}_{2}$ and ns- $\mathrm{ZrO}_{2}$ films deposited using different carrier gases. A linear fit in log-log scale highlights the power law character of the roughness evolution with film thickness (deposition time). Despite the difference in materials and deposition conditions, all films obey the same scaling law, compatible with the ballistic deposition regime. Adapted with permission from Refs [8,17]. Copyright 2015-2016 AIP Publishing.

The process of formation of a nanostructured thin film by SCBD is therefore very simple: nanoscale precursors with a broad size distribution are formed, then accelerated by a supersonic expansion against a substrate and assembled into a rough, disordered film, in a ballistic deposition regime..$^{8,17,20,26}$

The randomness of the interface obeys simple laws; the growth process is selfregulated, which makes it truly bottom-up. An example of the evolution of the surface nanoscale morphology of a nanostructured titania film is shown in Figures 3 A,B: controlling the film thickness, it is possible to precisely control the surface RMS roughness. 


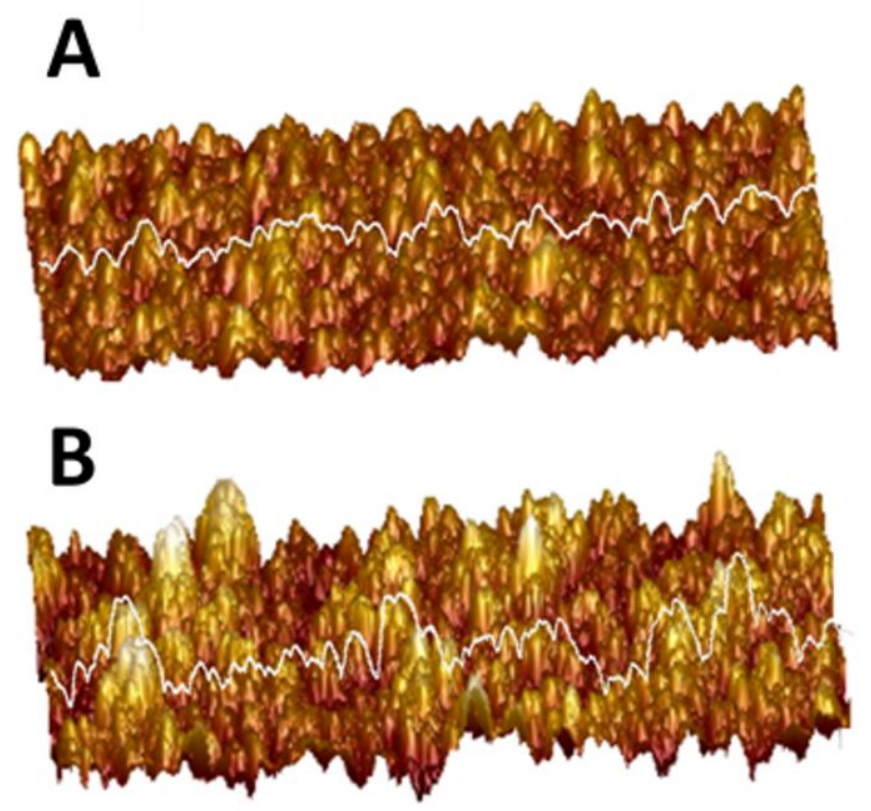

Figure 3. Representative $2 \mu \mathrm{m} \times 1 \mu \mathrm{m}$ topographic maps of $\mathrm{ns}-\mathrm{TiO}_{2}$ films of increasing roughness, demonstrating how surface corrugation evolves with increasing film thickness (deposition time). RMS roughness changes from $10 \mathrm{~nm}$ to $22 \mathrm{~nm}$ when the film thickness changes from about (A) $20 \mathrm{~nm}$ to (B) $100 \mathrm{~nm}$, respectively. The vertical scale range for all images is $100 \mathrm{~nm}$. Adapted with permission from Ref. [8]. Copyright 2015 AIP Publishing.

The evolution of cluster-assembled film morphology depends on the precursor cluster mass distribution. A quantitative demonstration is provided in Figure 2, showing the impact on RMS roughness of two different mass distributions of the precursors (see the inset of Figure 1), obtained by changing the gas where the cluster formation takes place prior to the supersonic expansion: ${ }^{8}$ for similar values of the film thickness, a marked difference in surface roughness is observed. Figure 3 also clearly shows the granular, nanoporous nature of cluster-assembled materials and the remarkable gain in specific area due to the use of small nanometer-sized building blocks. The surface pores, surface specific area, as well as the RMS roughness, 
depend on film thickness, ${ }^{8,13,17}$ and increase with it. As the film thickness increases, not only the surface becomes rougher, but also the average lateral dimension of the largest morphological features (the correlation length $\xi$ ) increases. An evolution of the aspect ratio of surface pores is therefore present. The increase of surface roughness $\mathrm{w}$ determines the evolution of surface asperities (their height, area, and surface density change), ${ }^{18,27}$ but also the increase of the number of pores having higher aspect ratio, as well as the average pore volume. ${ }^{13}$

\section{INFLUENCE OF THE SURFACE NANOSCALE MORPHOLOGY ON FUNCTIONAL INTERFACIAL PROPERTIES}

\section{Surface charge density in water and the isoelectric point}

The value of the $\mathrm{pH}$ at which the net surface charge is zero, the isoelectric point (IEP), ${ }^{28}$ plays an important role in the interaction of the surface with proteins. ${ }^{29}$ The electrical double layer properties depends on the characteristic lengths of the system (typically on their ratios): the Debye screening length $\lambda_{D}$ of the electrolyte, the surface RMS roughness $\mathrm{w}$ and the correlation length $\xi$ and, on a local scale, pores width and depth. ${ }^{8,27} \mathrm{We}$ demonstrated that by controlling the nanoscale morphology of cluster-assembled surfaces, it is possible to tune these ratios and explore a variety of interaction regimes. For instance, we have observed a remarkable reduction of the IEP by several $\mathrm{pH}$ units on rough ns- $\mathrm{TiO}_{2}$ nanostructured surfaces, with respect to flat crystalline rutile $\mathrm{TiO}_{2}{ }^{27}$

We recently proposed that the roughness-induced self-overlap of the electrical double layers can be responsible for deviations from the trend expected for flat surfaces, ${ }^{27}$ where the IEP is not expected to change. Nanostructured surfaces offer 
indeed the unique possibility of inducing self-overlap effects between adjacent regions of the same surface, due to the abundance of high aspect-ratio nanoscale regions providing extreme confinement to the electrolyte. Locally, the self-overlap effect can be dramatic, because the pores with nearly nanometer and sub-nanometer dimensions are smaller than the Debye length; this can lead to severe chargeregulation phenomena and strong deviations from the linearized Poisson-Boltzmann theory. ${ }^{30}$ This in turn may have an impact on the IEP, although the exact mechanisms are still unclear. Further work, both theoretical and experimental, is needed to get some deeper insight on this phenomenon.

The remarkable perturbations of the electrostatic interfacial double layer observed on cluster-assembled surfaces are expected to influence the interfacial adsorption/interaction of proteins and biologically relevant nano-objects.

\section{Wettability of cluster-assembled surfaces}

Surface morphology and surface charge have an important impact on the wetting behavior of nanostructured films. ${ }^{22,31-33}$ Surface wettability, in turn, can strongly affect the properties of biotic/abiotic interfaces and their interactions with proteins, enzymes and cells. ${ }^{34,35}$ We have shown that by combining the control of surface morphology to the control of surface chemistry of ns- $\mathrm{TiO}_{2}$ films, it is possible to tune the contact angle from $0^{\circ}$ (complete wetting) to $140^{\circ}$ (at the onset of superhydrophobicity). ${ }^{22}$ In particular, by applying simple thermal treatments in ambient atmosphere it is possible to switch from hydrophobicity to hydrophilicity and viceversa. Remarkably, by controlling surface roughness in the typical range 3-30 nm, it is possible to finely tune the contact angle within a given wetting regime. We have observed a similar scaling of the contact angle with roughness for nanostructured 
carbon films deposited by $\mathrm{SCBD} .^{31}$ An important factor that can provide an enhancement of hydrophobicity of rough surfaces is the formation of air pockets, which is possible also in intrinsically hydrophilic surfaces provided some overhangs are present, at least at the smallest scales. ${ }^{32,36}$ This condition is certainly satisfied in nanoporous materials. Remarkably, the two main channels for controlling film wettability, surface morphology and thermal annealing, turned out to be independent from each other. Changes in wettability induced by thermal annealing are reversible, the complete wettability recovery occurring in ambient air within several hours, due to hydrocarbon contamination. The surface wetting properties are therefore stable in air on the typical time scales of experiments or further sample treatments.

\section{BIOLOGICALLY-RELEVANT PROPERTIES OF CLUSTER- ASSEMBLED NANOSTRUCTURED FILMS}

In order to assess how the nanoscale topography determines the interaction of biological entities with surfaces, our starting point was the observation that clusterassembled films (titania and zirconia) are very good substrates for the growth and adhesion of primary, cancer and stem cells without any chemical functionalization of the surface. ${ }^{37,38}$ These films possess, at the nanoscale, a granularity and porosity mimicking those of typical extracellular matrix structures and adsorption properties that favor surface interaction with the proteins present in the cell culture media or directly produced by the cells. ${ }^{13}$ Adsorption of proteins on artificial surfaces in contact with biological fluids (blood, serum, culture media, etc.) is the first step of the formation of biotic/abiotic interfaces. ${ }^{3}$ This aspect has been elaborated in deep detail for nanoparticles in biological media, by introducing the concept of protein corona. ${ }^{39}$ The formation of a protein corona modifies the surface of nanoparticles and therefore 
the interface behavior depends on the composition of the corona. A large number of studies has been recently published on the characterization of protein corona of nanoparticles; ${ }^{40}$ quite surprisingly, only relatively few studies have been dedicated to the characterization of protein adsorption on nanostructured material surfaces as the key phenomenon regulating the subsequent cell attachment and adhesion. ${ }^{41}$

The full characterization of the adsorption of proteins on a surface is challenging since the adsorption process is influenced by the interplay of the physicochemical properties of the surface, such as structure, topography, surface charge, hydrophilicity/hydrophobicity with the environmental factors, such as protein mass, concentration, isoelectric point and exposed epitopes. ${ }^{3}$

\section{Adsorption of proteins on cluster-assembled surfaces}

The results obtained on the protein corona of nanoparticles in solution cannot be directly transferred to nanostructured surfaces characterized by a completely different dependence of the surface energy from the surface topography and crystalline structure. ${ }^{13,17,22,27,42}$ The picture emerging from the reports about protein adsorption on nanostructured surfaces is quite contradictory: no influence of the nanoscale morphology is reported by some authors, ${ }^{43}$ while others show data indicating an increase of the amount of adsorbed proteins with the increase of nanoscale surface roughness. ${ }^{44}$ These incoherent observations can be partially understood by considering the lack of any quantitative characterization of protein adsorption on nanostructured surfaces, due to the difficulty of establishing suitable experimental tools to control the large number of parameters affecting the adsorption process. $^{41}$ 
By exploiting the quantitative control on nanoscale roughness provided by SCBD, we performed a full characterization of protein adsorption elucidating also the role of protein concentration. ${ }^{13}$

We developed a quantitative, high-throughput method to conduct a parallel analysis of the protein-surface interaction, while systematically varying surface roughness, protein concentration and protein type. Our high-throughput approach is based on protein-surface interaction microarrays (PSIM) designed to yield protein adsorption isotherms for a panel of proteins on several surfaces simultaneously (Figure 4A). ${ }^{13,41}$ With PSIM we demonstrated the simultaneous characterization of 1,200 protein-surface interactions obtaining the protein adsorption isotherms on nanostructured surfaces with controlled topography ${ }^{13,41}$ (Figures 4 B,C).

We showed that the Langmuir isotherm model adequately reproduces our experimental data for all the tested proteins and that the surface nanoscale morphology drastically influences the amount of adsorbed proteins, in particular the saturation uptake increases non-linearly as nanoscale roughness increases. ${ }^{13}$ This indicates that the effect produced by increasing roughness is not justified by the simple increase of the surface specific area, nor by the creation of new adsorption sites. The measured saturation uptake is not directly proportional to the number of adsorption sites on the surface showing that the increase in protein adsorption is more than linear as a function of the increase of disposable adsorption space on the surface. ${ }^{13,45}$ 

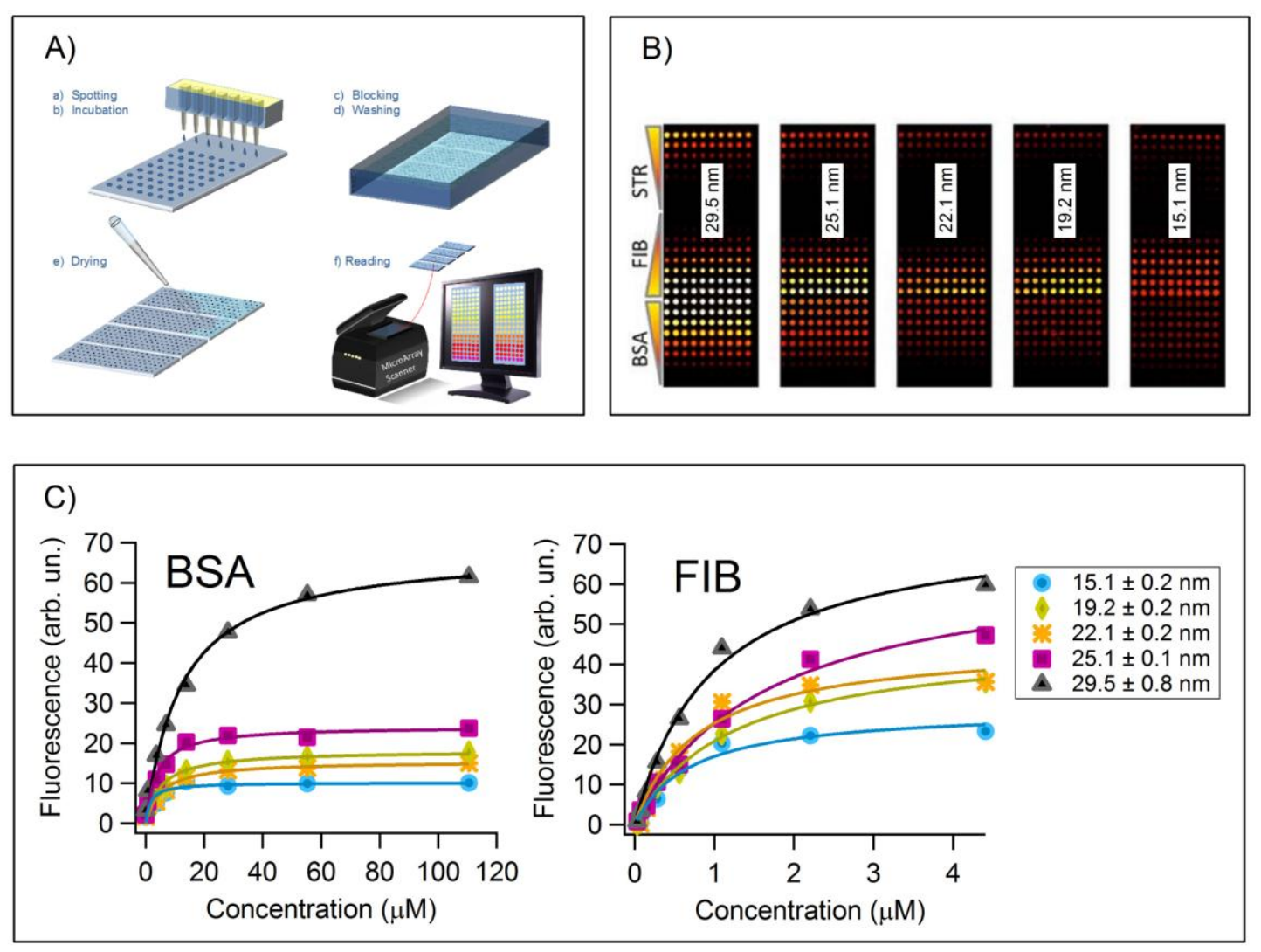

Figure 4. (A) Sketch of the PSIM protocol: spotting of droplets of labeled proteins diluted at different concentrations, incubation, blocking, washing, drying, and evaluation of the amount of adsorbed proteins with microarray scanner; (B) slides read-out of 8 concentrations of Bovine Serum Albumin (BSA), Fibrinogen (FIB) and Streptavidin (STR) spotted in 10 replica on 5 nanostructured substrates with different roughness (values are indicated on each slide); (C) adsorption data fitted with Langmuir isotherms of BSA and FIB as function of protein concentration for the 5 surfaces with different nanoscale roughness.

\section{Effect of nanotopography on cell differentiation}

The nanotopographical features of the extracellular microenvironment encountered by cells in vivo or in vitro have a fundamental impact on cellular properties and behavior, such as e.g. cell morphology, proliferation and differentiation. ${ }^{2,5,6}$ This impact is made possible by the cellular capability defined as mechanotransduction. Cells perceive mechanical and nanotopographical cues of the 
ECM and convert the information into modulations of the cytoskeletal organization and biomechanics, which are eventually translated via mechanosensitive signaling cascades and nuclear rearrangements into corresponding cellular responses (for a graphic illustration see the conspectus). ${ }^{5,6,46,47}$ Integrin adhesion complexes (IAC) are the interfacing key structures in this process. The eponymous transmembrane integrin proteins mediate extracellularly the 'sensing' and the adhesion to the ECM and connect it intracellularly via adaptor proteins to the actin cytoskeleton. The spatiotemporally dynamic composition and dimension of IAC comprise different hierarchic maturation grades, from nascent adhesions via focal complexes (FC) to focal adhesions (FA). The process of FA formation starts with the adaptor protein talin which couples actomyosin-generated forces to the integrins engaging a "molecular clutch" and allowing force transmission. In case of sufficient build-up of tension, talin is stretched and unfolded, enabling vinculin binding, which reinforces the structure. Vinculin, in turn, initiates a force-dependent recruitment of further IAC components leading to integrin clustering, stress fiber formation and the modulation of transmitted forces and signals. The maturation degree depends directly on the biophysical configuration (i.e. rigidity and nanotopography) of the ECM.,6,46,47,48 Nanotopographical features, such as e.g. the layout, area and height of asperities and the distance between adhesion sites, dictate IAC and cytoskeletal organization.,

Because of this essential function concerning perception and integration of nanoenvironmental signals, IAC are involved in virtually all developmental and physiological processes. ${ }^{5,6,46,47}$ However, fundamental aspects of the mechanotransductive signal integration still remain to be clarified due to the complexity and versatility of the involved components. ${ }^{47}$ 
To quantitatively address the influence of nanoscale topography on mechanotransduction, we exploit the capability of SCBD of large-scale production of substrates with controlled nanotopography. ${ }^{18}$

We focused our work primarily on a particularly interesting cell type: the neuronal one. Neuronal differentiation is accompanied by radical morphological and cytoskeletal rearrangements during the formation of neurites, dendrites and axons, largely regulated by IAC and mechanotransductive signaling. ${ }^{49,50}$ In PC12 cells, a broadly accepted model system for neuronal differentiation, we systematically traced how the cellular interaction with the nanotopography of cluster-assembled titania ${ }^{51}$ and zirconia films ${ }^{18}$ impacts on the mechanotransductive sequence and the differentiation behavior (Figure 5).
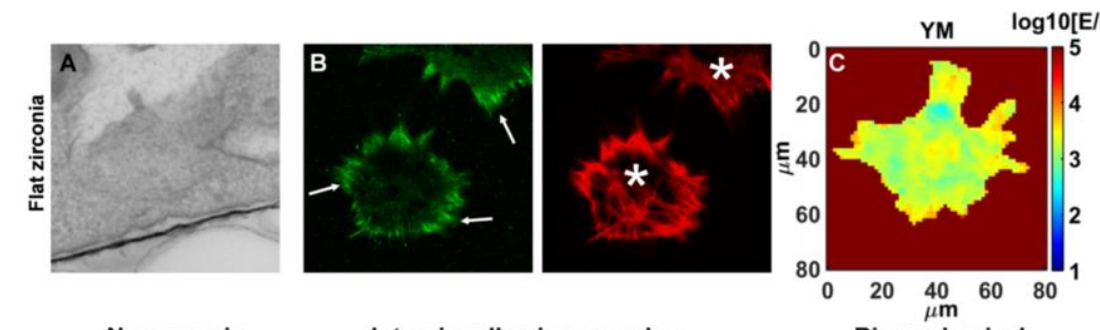

Biomechanical Integrin adhesion complex and cytoskeletal configuration

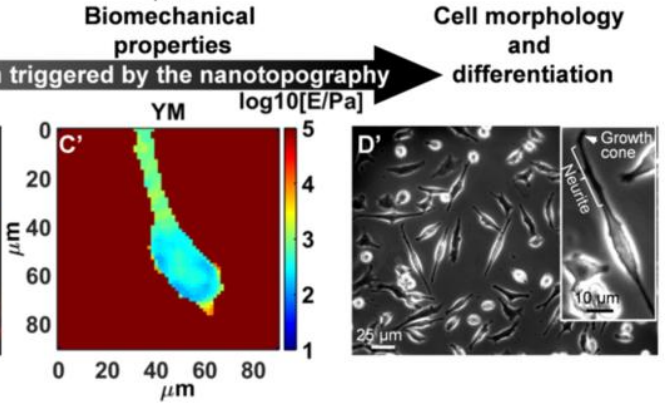

Figure 5. (A,A') The TEM images demonstrate the interface between PC12 cells and (A) flat zirconia (produced by E-beam evaporation) or (A') nanostructured zirconia surfaces (with $15 \mathrm{~nm}$ RMS roughness) fabricated by SCBD. Cells interact only with the upper part of the surface asperities. (B, B') The staining of vinculin (a crucial IAC component) recorded by TIRF microscopy show (B) FA formation (arrows) on flat zirconia, compared to (B') the smaller structures with FC dimensions (dashed arrows) on the nanostructured surface. On the right, the corresponding epifluorescence images of filamentous actin cytoskeleton are shown. 
The asterisks indicate zones of stress fiber formation on flat zirconia, not present on the nanostructured zirconia. (C, C') The Young's Modulus (YM) maps of the cellular elasticity on (C) flat or (C') nanostructured zirconia demonstrate lower cellular rigidity in the latter condition. (D, D') The phase contrast images display the effect of the mechanotransductive signaling on PC12 differentiation, (D') paralleled by morphological changes (neurite outgrowth) on the nanostructured surface (inset: typical morphology of differentiated PC12). (A-D) Adapted with permission from Ref. [18]. Copyright 2016 BioMed Central.

We showed that the spatial organization of the surface asperities obtained by SCBD presents nanotopographical cues to the cells ${ }^{18}$ that effectively mimic those found in physiological ECM. ${ }^{2}$ We recognized that appropriate nanotopographical surface features, by restricting integrin clustering and IAC maturation to the FC level, alter the IAC composition and dynamics, the cytoskeletal organization, and the nanomechanical cellular properties, compared to the featureless flat surfaces (Figure 5A-C). Consequentially, these nanotopography-triggered effects on the mechanotransductive machinery ${ }^{18}$ promote differentiative events, such as the induction of NO, ERK1/2 $2^{51}$ and CREB signaling cascades, which eventually leads to neuritogenesis (Figure 5D') and a drastic change in the cellular program (Figure 6) of the neuron-like PC12. ${ }^{18,51}$ Congruently, we found that the nanotopography of clusterassembled zirconia surfaces foster maturation, neurito-/synaptogenesis and neural network formation of primary hippocampal neurons involving mechanotransductive pathways. ${ }^{19}$ Our results suggest that the effects of suitable nanotopographies, by acting directly on IAC nanoarchitecture and dynamics, can be similar to those of soft substrates, either at the IAC level and regarding neurogenic differentiation. ${ }^{48,52}$ 


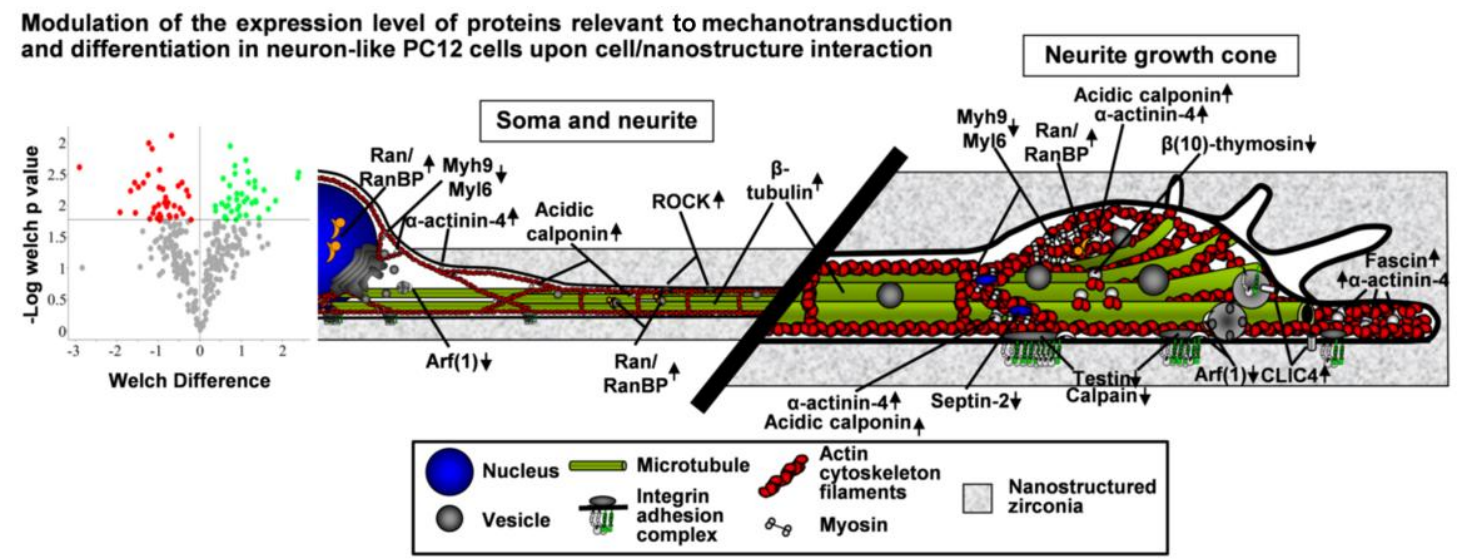

Figure 6. Proteomics allowed studying changes of the cellular program triggered by the cell/nanotopography interaction. The volcano plot on the left displays the identified differentially expressed proteins upon 24 hours of PC12 cell/nanostructure interaction, compared to flat zirconia. The colored dots indicate the (green) up- or (red) downregulated proteins. The cartoon illustrates a section of a differentiated neuronal cells with a close-up of the neurite growth cone (compare also Figure 5D') depicting examples of proteins (arrows show up- or downregulation with respect to flat zirconia) with functions relevant to mechanotransduction and neuronal differentiation. Adapted with permission from Ref. [18]. Copyright 2016 BioMed Central.

\section{SUMMARY AND PERSPECTIVES}

We described our approach to quantitatively understand and therewith control the physical and biologically-relevant properties that influence biotic/abiotic interactions at nanostructured surfaces. We showed that surfaces with controlled topography at the nanoscale can be efficiently fabricated by supersonic cluster beam deposition. The ballistic deposition regime in which the growth is taking place assures the evolution of the nanoscale features according to simple and universal scaling laws.

Our work, systematically conducted over more than one decade, demonstrates that structural and functional properties of the cluster-assembled surfaces relevant to their biocompatibility can be engineered and efficiently reproduced. Specifically the 
isoelectric point, which plays a fundamental role in the adhesion of biological moieties, decreases as a function of the roughness. Moreover, the wettability turned out to be easily adjustable by controlling the surface roughness and by thermal treatments.

We developed a high-throughput method to determine quantitatively the protein adsorption capacity of the nanostructured surfaces, showing that the adsorption is beyond the expected rise proportional to the growing specific surface area with an almost linear decrease of the protein binding affinity.

Another very important outcome of our studies is the demonstration that the spatial organization of the surface asperities has an impact on mechanotransductive signaling of cells by the modulation of important cellular adhesion-related parameters, such as integrin clustering and focal adhesion maturation, and the correlated biomechanics. This eventually leads to a change in the cellular program and differentiation, in particular shown for neuronal cells.

These results provide overall a deep insight on how the surface nanoscale structure influences the interaction with biological entities. This constitutes the necessary foundation for efficient near- to midterm future exploitation of SCBD for versatile bio-applications. After precise dissection of the appropriate biophysical cues, the cluster-assembled surfaces can be integrated into cell culture devices promoting desired cellular responses, e.g. differentiation of primary or stem cells applicable in cell replacement strategies. In the contest of neuronal cells, this already shown potential could be the basis for the design of enhanced neural interfaces and circuits.

\section{ACKNOWLEDGEMENTS}

We thank F. Borghi for her contribution to AFM analysis. 


\section{AUTHOR INFORMATION \\ Corresponding author}

*E-mail: paolo.milani@mi.infn.it

\section{Notes}

The authors declare no competing financial interest.

\section{Biographical information}

Cristina Lenardi is Associate Professor at the Department of Physics of the University of Milan (Italy). Her research areas concern the electronic characterization of nanostructured surfaces by photoelectron spectroscopy (XPS, UPS, etc.) and the microfabrication with novel polymeric materials for regenerative medicine and soft robotics. ResearcherID: http://www.researcherid.com/rid/J-5872-2012.

Paolo Milani is Full Professor at the Department of Physics of the University of Milan. He serves as Director of the Interdisciplinary Centre for Nanostructured Materials and Interfaces (CIMAINA). His research focuses on cluster-assembled materials and their integration on microfabricated platforms by exploiting the supersonic cluster beam deposition technology. He is active in the field of nanostructured systems and devices for stretchable electronics and optics, biotechnology and medicine, energy production and storage.

Alessandro Podestà is Associate Professor at the Department of Physics of the University of Milan. His research activity is focused on the investigation of systems and interfaces at the nanoscale by scanning probe techniques, and is supported by the 
development and implementation of atomic force microscopy -based techniques and protocols. ResearcherID: http://www.researcherid.com/rid/E-6568-2010.

Carsten Schulte is senior postdoc at CIMAINA, University of Milan. He received his Ph.D. in Biology from the University of Cologne. His research has been primarily concentrated on the interaction of cells with their microenvironment and the impact of the corresponding mechanotransductive signaling on cellular functioning and behavior. ORCID: http://orcid.org/0000-0002-7554-5342.

Gabriella Tedeschi is Associate Professor of Biochemistry at the University of Milan. Her researches deals with the characterization of proteins using classical enzymology, protein chemistry and proteomic approaches such as two-dimensional electrophoresis, immunochemistry and mass spectrometry, with applications ranging from the study of intact proteins and protein complexes to the characterization of complete proteomes and post-translational modifications of proteins from various biological sources. 


\section{REFERENCES}

(1) Mendes, P. M. Cellular Nanotechnology: Making Biological Interfaces Smarter. Chem. Soc. Rev. 2013, 42 (24), 9207.

(2) Gasiorowski, J. Z.; Murphy, C. J.; Nealey, P. F. Biophysical Cues and Cell Behavior: The Big Impact of Little Things. Annu. Rev. Biomed. Eng. 2013, 15 (1), 155-176.

(3) Vogler, E. A. Protein Adsorption in Three Dimensions. Biomaterials 2012, 33 (5), 1201-1237.

(4) Tenzer, S.; Docter, D.; Kuharev, J.; Musyanovych, A.; Fetz, V.; Hecht, R.; Schlenk, F.; Fischer, D.; Kiouptsi, K.; Reinhardt, C.; Landfester, K.; Schild, H.; Maskos, M.; Knauer, S. K.; Stauber, R. H. Rapid Formation of Plasma Protein Corona Critically Affects Nanoparticle Pathophysiology. Nat. Nanotechnol. 2013, 8 (10), 772-781.

(5) Dalby, M. J.; Gadegaard, N.; Oreffo, R. O. C. Harnessing Nanotopography and Integrin-matrix Interactions to Influence Stem Cell Fate. Nat. Mater. 2014, 13 (6), 558-569.

(6) Chen, W.; Shao, Y.; Li, X.; Zhao, G.; Fu, J. Nanotopographical Surfaces for Stem Cell Fate Control: Engineering Mechanobiology from the Bottom. Nano Today 2014, 9 (6), 759-784.

(7) Moon, S. K.; Kwon, J. S.; Baik, S. W.; Jeon, G. R.; Ro, J. H.; Eom, T. G.; Kim, K. N. Surface Characteristics of Nanostructure Formed on Sand Blasted with Large Grit and Acid Etched Dental Implant. Adv. Mater. Res. 2013, 647, 80-87.

(8) Podestà, A.; Borghi, F.; Indrieri, M.; Bovio, S.; Piazzoni, C.; Milani, P. Nanomanufacturing of Titania Interfaces with Controlled Structural and Functional Properties by Supersonic Cluster Beam Deposition. J. Appl. Phys. 2015, 118 (23), 234309.

(9) Manicone, P. F.; Rossi Iommetti, P.; Raffaelli, L. An Overview of Zirconia Ceramics: Basic Properties and Clinical Applications. J. Dent. 2007, 35 (11), 819826.

(10) Fei Yin, Z.; Wu, L.; Gui Yang, H.; Hua Su, Y. Recent Progress in Biomedical Applications of Titanium Dioxide. Phys. Chem. Chem. Phys. 2013, 15 (14), 4844.

(11) Heimann, R. B.; Lehmann, H. D. Bioceramic Coatings for Medical Implants: Trends and Techniques; 2015.

(12) Carbone, R.; De Marni, M.; Zanardi, A.; Vinati, S.; Barborini, E.; Fornasari, L.; Milani, P. Characterization of Cluster-Assembled Nanostructured Titanium Oxide Coatings as Substrates for Protein Arrays. Anal. Biochem. 2009, 394 (1), 712.

(13) Scopelliti, Pasquale Emanuele; Borgonovo, A.; Indrieri, M.; Giorgetti, L.; Bongiorno, G.; Carbone, R.; Podesta, A.; Milani, P. The Effect of Surface Nanometre-Scale Morphology on Protein Adsorption. PLOS ONE 2010, 5 (7), e11862.

(14) Wegner, K.; Piseri, P.; Tafreshi, H. V.; Milani, P. Cluster Beam Deposition: A Tool for Nanoscale Science and Technology. J. Phys. Appl. Phys. 2006, 39 (22), R439-R459.

(15) Barborini, E.; Kholmanov, I. N.; Piseri, P.; Ducati, C.; Bottani, C. E.; Milani, P. Engineering the Nanocrystalline Structure of TiO [sub 2] Films by Aerodynamically Filtered Cluster Deposition. Appl. Phys. Lett. 2002, 81 (16), 3052 . 
(16) Piseri, P.; Tafreshi, H. V.; Milani, P. Manipulation of Nanoparticles in Supersonic Beams for the Production of Nanostructured Materials. Curr. Opin. Solid State Mater. Sci. 2004, 8 (3-4), 195-202.

(17) Borghi, F.; Sogne, E.; Lenardi, C.; Podestà, A.; Merlini, M.; Ducati, C.; Milani, P. Cluster-Assembled Cubic Zirconia Films with Tunable and Stable Nanoscale Morphology against Thermal Annealing. J. Appl. Phys. 2016, 120 (5), 055302. (18) Schulte, C.; Rodighiero, S.; Cappelluti, M. A.; Puricelli, L.; Maffioli, E.; Borghi, F.; Negri, A.; Sogne, E.; Galluzzi, M.; Piazzoni, C.; Tamplenizza, M.; Podestà, A.; Tedeschi, G.; Lenardi, C.; Milani, P. Conversion of Nanoscale Topographical Information of Cluster-Assembled Zirconia Surfaces into Mechanotransductive Events Promotes Neuronal Differentiation. J. Nanobiotechnology 2016, 14 (1). (19) Schulte, C.; Ripamonti, M.; Maffioli, E.; Cappelluti, M.; Nonnis, S.; Puricelli, L.; Lamanna, J.; Piazzoni, C.; Podestà, A.; Lenardi, C.; Tedeschi, G.; Malgaroli, A.; Milani, P. Scale Invariant Disordered Nanotopography Promotes Hippocampal Neuron Development and Maturation with Involvement of Mechanotransductive Pathways. Front. Cell. Neurosci. 2016, 10, 267.

(20) Barabási, A.-L.; Stanley, H. E. Fractal Concepts in Surface Growth; Cambridge University Press: Cambridge, 1995.

(21) Dynamics of Fractal Surfaces; Family, F., Vicsek, T., Eds.; World Scientific: Singapore ; River Edge, N.J, 1991.

(22) Podesta, A.; Bongiorno, G.; Scopelliti, P. E.; Bovio, S.; Milani, P.; Semprebon, C.; Mistura, G. Cluster-Assembled Nanostructured Titanium Oxide Films with Tailored Wettability. J. Phys. Chem. C 2009, 113 (42), 18264-18269.

(23) Piseri, P.; Podesta, A.; Barborini, E.; Milani, P. Production and Characterization of Highly Intense and Collimated Cluster Beams by Inertial Focusing in Supersonic Expansions. Rev. Sci. Instrum. 2001, 72 (5), 2261-2267. (24) Castelnovo, C.; Podestà, A.; Piseri, P.; Milani, P. Fractal Analysis of Sampled Profiles: Systematic Study. Phys. Rev. E 2002, 65 (2), 021601.

(25) Robbins, M. O.; Cieplak, M.; Ji, H.; Koiller, B.; Martys, N. Growth in Systems with Quenched Disorder. In Growth Patterns in Physical Sciences and Biology; Garcia-Ruiz, J. M., Louis, E., Meakin, P., Sander, L. M., Eds.; Springer US: Boston, MA, 1993; Vol. 304, pp 65-75.

(26) Family, F.; Vicsek, T. Scaling of the Active Zone in the Eden Process on Percolation Networks and the Ballistic Deposition Model. J. Phys. Math. Gen. 1985, 18 (2), L75-L81.

(27) Borghi, F.; Vyas, V.; Podestà, A.; Milani, P. Nanoscale Roughness and Morphology Affect the IsoElectric Point of Titania Surfaces. PLoS ONE 2013, 8 (7), e68655.

(28) Delgado, A. V.; González-Caballero, F.; Hunter, R. J.; Koopal, L. K.; Lyklema, J. Measurement and Interpretation of Electrokinetic Phenomena. J. Colloid Interface Sci. 2007, 309 (2), 194-224.

(29) Elter, P.; Lange, R.; Beck, U. Electrostatic and Dispersion Interactions during Protein Adsorption on Topographic Nanostructures. Langmuir 2011, 27 (14), 8767-8775.

(30) Lyklema, J.; Duval, J. F. L. Hetero-Interaction between Gouy-Stern Double Layers: Charge and Potential Regulation. Adv. Colloid Interface Sci. 2005, 114$115,27-45$. 
(31) Ostrovskaya, L.; Podesta, A.; Milani, P.; Ralchenko, V. Influence of Surface Morphology on the Wettability of Cluster-Assembled Carbon Films. Europhys. Lett. 2003, 63 (3), 401-407.

(32) Herminghaus, S. Roughness-Induced Non-Wetting. Europhys. Lett. 2000, 52 (2), 165-170.

(33) Puah, L. S.; Sedev, R.; Fornasiero, D.; Ralston, J.; Blake, T. Influence of Surface Charge on Wetting Kinetics. Langmuir 2010, 26 (22), 17218-17224.

(34) Bodhak, S.; Bose, S.; Bandyopadhyay, A. Role of Surface Charge and Wettability on Early Stage Mineralization and Bone Cell-materials Interactions of Polarized Hydroxyapatite. Acta Biomater. 2009, 5 (6), 2178-2188.

(35) Arima, Y.; Iwata, H. Effect of Wettability and Surface Functional Groups on Protein Adsorption and Cell Adhesion Using Well-Defined Mixed Self-Assembled Monolayers. Biomaterials 2007, 28 (20), 3074-3082.

(36) Cassie, A. B. D.; Baxter, S. Wettability of Porous Surfaces. Trans. Faraday Soc. 1944, 40, 546.

(37) Belicchi, M.; Erratico, S.; Razini, P.; Meregalli, M.; Cattaneo, A.; Jacchetti, E.; Farini, A.; Villa, C.; Bresolin, N.; Porretti, L.; Lenardi, C.; Milani, P.; Torrente, Y. Ex Vivo Expansion of Human Circulating Myogenic Progenitors on ClusterAssembled Nanostructured TiO2. Biomaterials 2010, 31 (20), 5385-5396.

(38) Carbone, R.; Marangi, I.; Zanardi, A.; Giorgetti, L.; Chierici, E.; Berlanda, G.; Podesta, A.; Fiorentini, F.; Bongiorno, G.; Piseri, P.; Pelicci, P.; Milani, P.

Biocompatibility of Cluster-Assembled Nanostructured TiO2 with Primary and Cancer Cells. BIOMATERIALS 2006, 27 (17), 3221-3229.

(39) Cedervall, T.; Lynch, I.; Lindman, S.; Berggard, T.; Thulin, E.; Nilsson, H.; Dawson, K. A.; Linse, S. Understanding the Nanoparticle-Protein Corona Using Methods to Quantify Exchange Rates and Affinities of Proteins for Nanoparticles. Proc. Natl. Acad. Sci. 2007, 104 (7), 2050-2055.

(40) Rahman, M.; Laurent, S.; Tawil, N.; Yahia, L.; Mahmoudi, M. Nanoparticle and Protein Corona. In Protein-Nanoparticle Interactions; Springer Berlin Heidelberg: Berlin, Heidelberg, 2013; Vol. 15, pp 21-44.

(41) Scopelliti, P. E.; Bongiorno, G.; Milani, P. High Throughput Tools for the Study of Protein-Nanostructured Surface Interaction. Comb. Chem. High Throughput Screen. 2011, 14 (3), 206-216.

(42) Singh, A. V.; Ferri, M.; Tamplenizza, M.; Borghi, F.; Divitini, G.; Ducati, C.; Lenardi, C.; Piazzoni, C.; Merlini, M.; Podestà, A.; Milani, P. Bottom-up Engineering of the Surface Roughness of Nanostructured Cubic Zirconia to Control Cell Adhesion. Nanotechnology 2012, 23 (47), 475101.

(43) Han, M.; Sethuraman, A.; Kane, R. S.; Belfort, G. Nanometer-Scale Roughness Having Little Effect on the Amount or Structure of Adsorbed Protein. Langmuir 2003, 19 (23), 9868-9872.

(44) Rechendorff, K.; Hovgaard, M. B.; Foss, M.; Zhdanov, V. P.; Besenbacher, F. Enhancement of Protein Adsorption Induced by Surface Roughness. Langmuir 2006, 22 (26), 10885-10888.

(45) Gailite, L.; Scopelliti, P. E.; Sharma, V. K.; Indrieri, M.; Podestà, A.; Tedeschi, G.; Milani, P. Nanoscale Roughness Affects the Activity of Enzymes Adsorbed on Cluster-Assembled Titania Films. Langmuir 2014, 30 (20), 5973-5981.

(46) Wang, N.; Tytell, J. D.; Ingber, D. E. Mechanotransduction at a Distance: Mechanically Coupling the Extracellular Matrix with the Nucleus. Nat. Rev. Mol. Cell Biol. 2009, 10 (1), 75-82. 
(47) Humphries, J. D.; Paul, N. R.; Humphries, M. J.; Morgan, M. R. Emerging Properties of Adhesion Complexes: What Are They and What Do They Do? Trends Cell Biol. 2015, 25 (7), 388-397.

(48) Elosegui-Artola, A.; Oria, R.; Chen, Y.; Kosmalska, A.; Pérez-González, C.; Castro, N.; Zhu, C.; Trepat, X.; Roca-Cusachs, P. Mechanical Regulation of a Molecular Clutch Defines Force Transmission and Transduction in Response to Matrix Rigidity. Nat. Cell Biol. 2016, 18 (5), 540-548.

(49) Kerstein, P. C.; Nichol IV, R. H.; Gomez, T. M. Mechanochemical Regulation of Growth Cone Motility. Front. Cell. Neurosci. 2015, 9.

(50) Myers, J. P.; Santiago-Medina, M.; Gomez, T. M. Regulation of Axonal Outgrowth and Pathfinding by Integrin-Ecm Interactions. Dev. Neurobiol. 2011, 71 (11), 901-923.

(51) Tamplenizza, M.; Lenardi, C.; Maffioli, E.; Nonnis, S.; Negri, A.; Forti, S.; Sogne, E.; De Astis, S.; Matteoli, M.; Schulte, C.; Milani, P.; Tedeschi, G. Nitric Oxide Synthase Mediates PC12 Differentiation Induced by the Surface Topography of Nanostructured TiO2. J. Nanobiotechnology 2013, 11 (1), 35.

(52) Franze, K.; Janmey, P. A.; Guck, J. Mechanics in Neuronal Development and Repair. Annu. Rev. Biomed. Eng. 2013, 15 (1), 227-251. 